\title{
Prototype of extracorporeal membrane oxygenation (ECMO) therapy simulator used in regional ECMO program
}

\author{
Mateusz Puslecki ${ }^{1,2,3}$, Marcin Ligowski ${ }^{2,3}$, Michal Kiel ${ }^{4}$, Marek Dabrowski, ${ }^{1,5}$, Sebastian Stefaniak ${ }^{2,3}$, \\ Maciej Sip ${ }^{1,5}$, Adrian Maciejewski ${ }^{1}$, Agata Dabrowska ${ }^{1,5}$, Ilona Kiel-Puslecka ${ }^{6}$, Tomasz Kłosiewicz ${ }^{1}$, \\ Marcin Misterski ${ }^{2,3}$, Piotr Buczkowski ${ }^{2,3}$, Lukasz Szarpak ${ }^{7,8}$, Kurt Ruetzler ${ }^{9}$, Bartlomiej Perek ${ }^{2,3}$, Michael \\ Czekajlo $^{5,10,11}$, Marek Jemielity ${ }^{2,3}$
}

${ }^{1}$ Department of Medical Rescue, Poznan University of Medical Sciences, Poznan, Poland; ${ }^{2}$ Department of Cardiac Surgery and Transplantology, Poznan University of Medical Sciences, Poznan, Poland; ${ }^{3}$ Clinical Hospital SKPP, Poznan, Poland; ${ }^{4}$ IT WORKS, Wroclaw, Poland; ${ }^{5}$ Polish Society of Medical Simulation, Poznan, Poland; ${ }^{6}$ Department of Palliative Medicine, Poznan University of Medical Sciences, Poznan, Poland; ${ }^{7}$ Department of Emergency Medicine, Medical University of Warsaw, Warsaw, Poland; ${ }^{8}$ Lazarski University, Warsaw, Poland; ${ }^{9}$ Departments of Outcomes Research and General Anesthesiology, Cleveland Clinic, Cleveland, Ohio, USA; ${ }^{10}$ Department of Surgery, Hunter Holmes McGuire VA Medical Center, Richmond, USA; ${ }^{11}$ Lublin Medical University, Medical Simulation Center, Lublin, Poland

Contributions: (I) Conception and design: M Pusleck, M Ligowski, M Kiel, M Dabrowski, S Stefaniak, M Sip, A Maciejewski; (II) Administrative support: None; (III) Provision of study materials or patients: None; (IV) Collection and assembly of data: None; (V) Data analysis and interpretation: None X; (VI) Manuscript writing: All authors; (VII) Final approval of manuscript: All authors.

Correspondence to: Lukasz Szarpak. Lazarski University, 43 Swieradowska Str, Warsaw 02-662, Poland. Email: Lukasz.szarpak@gmail.com.

Background: Simulation is widely accepted as an important tool in training and educating healthcare providers. The first regional polish extracorporeal membrane oxygenation (ECMO) program called "ECMO for Greater Poland" was recently started.

Methods: We present a prototype for ECMO prepared for high-fidelity medical simulation in extracorporeal life support. ECMO therapy is a complex, difficult and expensive therapy in patient care. We have constructed an advanced ECMO simulation prototype, which can be used as a training tool in scenarios that prepare for real-life experiences. The "ECMO for Greater Poland" program uses high-fidelity simulation as a superior tool to simulate several complex clinical scenarios, and consequently train healthcare providers in rare, complicated and expensive procedures. The training course is standardized and allows repeatable training, improvement of skills, and an objective verification of trained skills.

Results: The ECMO simulation prototype is designed to replicate the physiological circulatory system and simulate several scenarios (i.e., bleeding, low pressure, occlusion, reaction for proper and incorrect pharmacological treatment). The electronic core control unit (CCU) with silicone tubes, artificial vessels (modified polyethylene) and analog components can be connected to an ECMO machine to emulate the human body during an ECMO simulation. If necessary, damaged and used parts can easily be replaced. The total cost of the simulator is approximately 450 and 50 USD for disposable parts.

Conclusions: This newly developed advanced ECMO simulation prototype was recently introduced into education and regularly training of healthcare providers of the "ECMO for Greater Poland" program. The simulation based training program is financially affordable and enables clinical teaching in a wider range of clinical scenarios. The ECMO simulation prototype consequently improves level of expertise of the healthcare providers and finally improves quality in patient care.

Keywords: Extracorporeal membrane oxygenation (ECMO); program; simulator; prototype

Submitted Feb 05, 2018. Accepted for publication Apr 18, 2018.

doi: $10.21037 /$ jtd.2018.07.25

View this article at: http://dx.doi.org/10.21037/jtd.2018.07.25 


\section{Introduction}

Extracorporeal membrane oxygenation (ECMO) is a complex and expensive complication-burdened life support modality in order to treat patients with respiratory and/ or circulatory failure. A group of ECMO enthusiasts recently launched the first polish ECMO program called "ECMO for Greater Poland". The program's objective is to take full advantage of the indications for extracorporeal life support for the 3.5 million inhabitants of the Greater Poland region $(1,2)$.

Simulation-based teaching and regularly training of healthcare providers is fundamental (3-5). High-fidelity simulation is used to simulate and train rare procedures and emergency situations and finally to familiarize healthcare providers with ECMO specific treatments and strategies (6). Different ECMO simulators have been used in the past, ranging from simple mannequin modifications to high-fidelity simulators (7-9). Commercially available ECMO simulators are mostly limited by the high costs, experimental status, and the impossibility to adapt the simulator to local special circumstances. Therefore, we aimed to develop an advanced ECMO simulator prototype, which can be incorporated in any commercially available full-body patient simulator.

\section{Methods}

The prototype is equipped with an electronic core control unit (CCU) (Figure 1), a set of synthetic valves, pressure sensors, and hydraulic pumps. The functions of the CCU is to stabilize the hydraulic system (flow of simulated blood, differential pressures in the arterial and venous lines), and providing real-time information's about the system to the provider via an audiovisual display. Consequently, the system is able to detect any clinical relevant physiological change or clinical deterioration and initiates adequate changes in clinical parameters including (i.e., bleeding, low blood pressure, occlusion, reaction for proper and incorrect pharmacological therapy).

Silicone tubes and artificial vessels (modified polyethylene) can be realistically cannulated using ultrasound. The tubes can be connected to an ECMO machine to emulate the human body during an ECMO run.

The CCU along with the silicone tubes, artificial vessels and electronic components can be integrated into any commercially available mannequin (Figures 1 and 2). The CCU hardware element includes both, digital and analog components that are controlled by a computer-based software connected to the CCU via a serial port (RS232). The software deliberately analyzes the measurements obtained from the electronic controllers modulating the sensors, control pumps and valves and visualizes the data for the provider. The electronic controllers influence the ECMO simulated blood circuit, and hence can be used as a tool to recreate various clinical scenarios.

The total cost of the first simulator prototype (CSM rev. 1.0) is approximately 450 USD and additionally about 50 USD for disposable parts. The hardware components consist of a PCB (printed circuit board) and a selection of essential electronic components. The software component was created on the basis of a code that supports high-fidelity simulation. The final element is the framework supporting the testing and validation of the ECMO simulator (Figure 3).

\section{Results}

ECMO team training "in situ" simulation scenarios have been created and preliminarily examined by specialists (Table 1). The simulation training center was adapted as an intensive care unit. The CardioHelp (Maquet, Getinge, Rastatt, Germany) circuit was connected to SimMan 3G manikin (Laerdal, Stavanger, Norway) via simulated venovenous cannulation. The patient simulator was intubated and connected to a respirator (Maquet, Getinge, Rastatt, Germany); and via controller to vital monitor (Laerdal).

During the first four months (Sep-Dec 2016) of our Program, the following high-fidelity medical simulation scenarios using the ECMO simulation prototype have been generated so far:

* "ECMO for DCD" which includes: prehospital identification, cardiopulmonary resuscitation (CPR) according to advanced life support (ALS), perfusion therapy (CPR-ECMO or DCD-ECMO), inclusion and exclusion criteria for organ donor program, automated chest compression (ACC), transport, DCD confirmation, donor authorization, venoarterial (VA) cannulation of a mannequin's artificial vessels and initiating organ perfusion on-site.

* "ECMO for intoxication" which includes: hospital identification (Department of Toxicology), poisoning treatment, CPR according to ALS, ACC, venoarterial cannulation for the implementation of ECMO therapy and transport to hospital (Department of Cardiac Surgery).

* "ECMO for RRF" (reversible respiratory failure) 

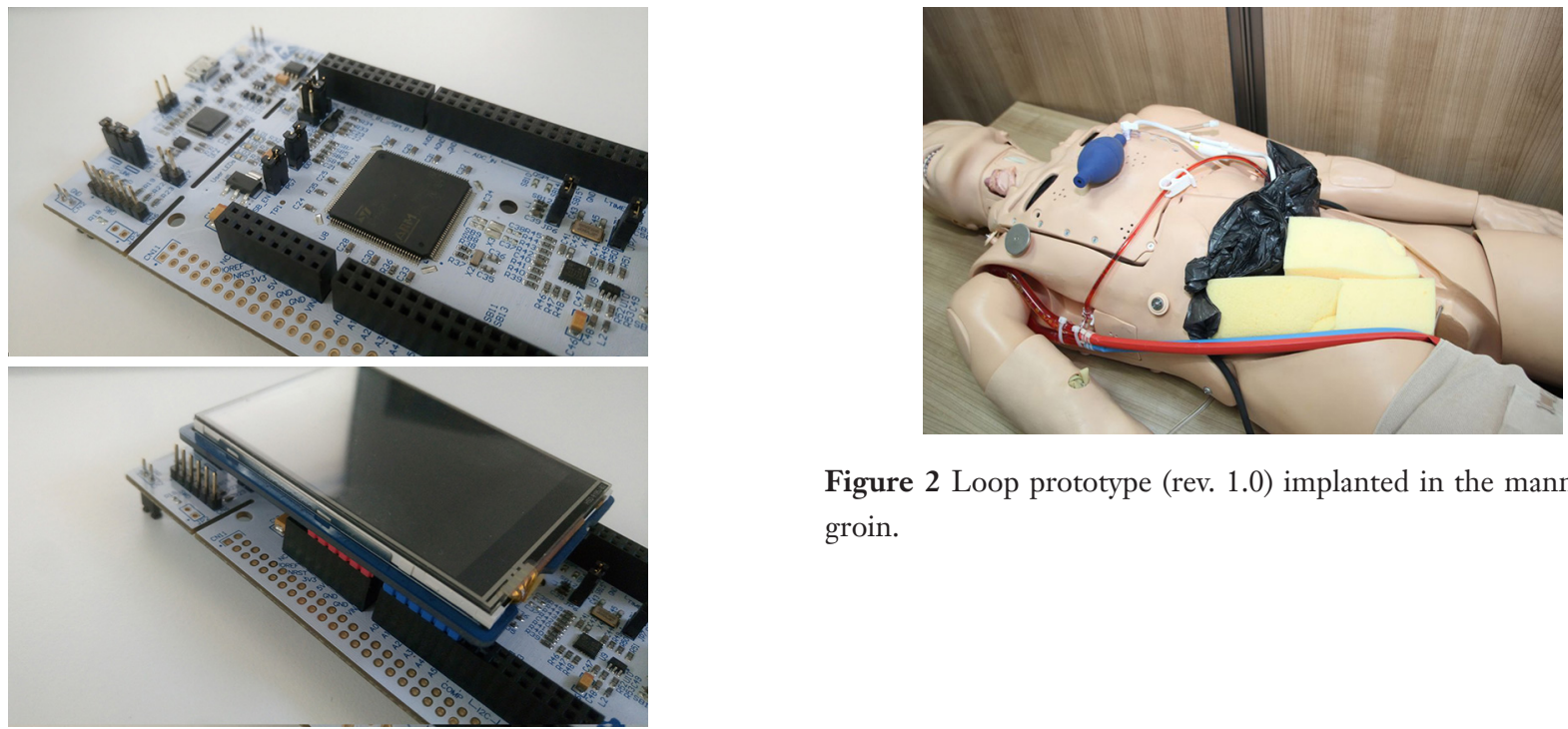

Figure 2 Loop prototype (rev. 1.0) implanted in the mannequin's groin.

Figure $1 \mathrm{CCU}$ prototype (rev. 2.0) w/o equipment. CCU, core control unit.

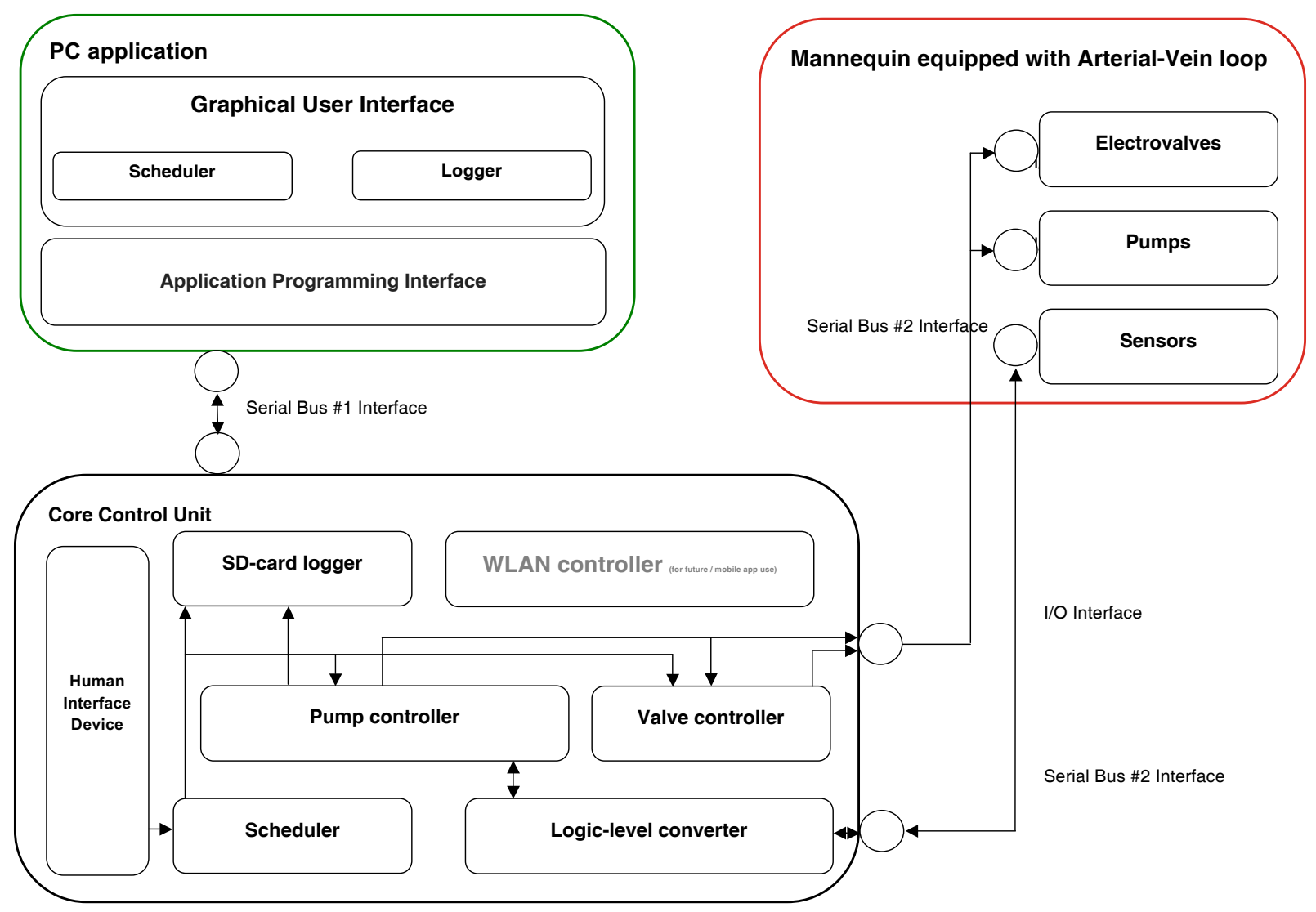

Figure 3 Prototype of the ECMO simulator architecture. ECMO, extracorporeal membrane oxygenation. 
Table 1 "In situ" 17 simulation scenarios for educational purposes

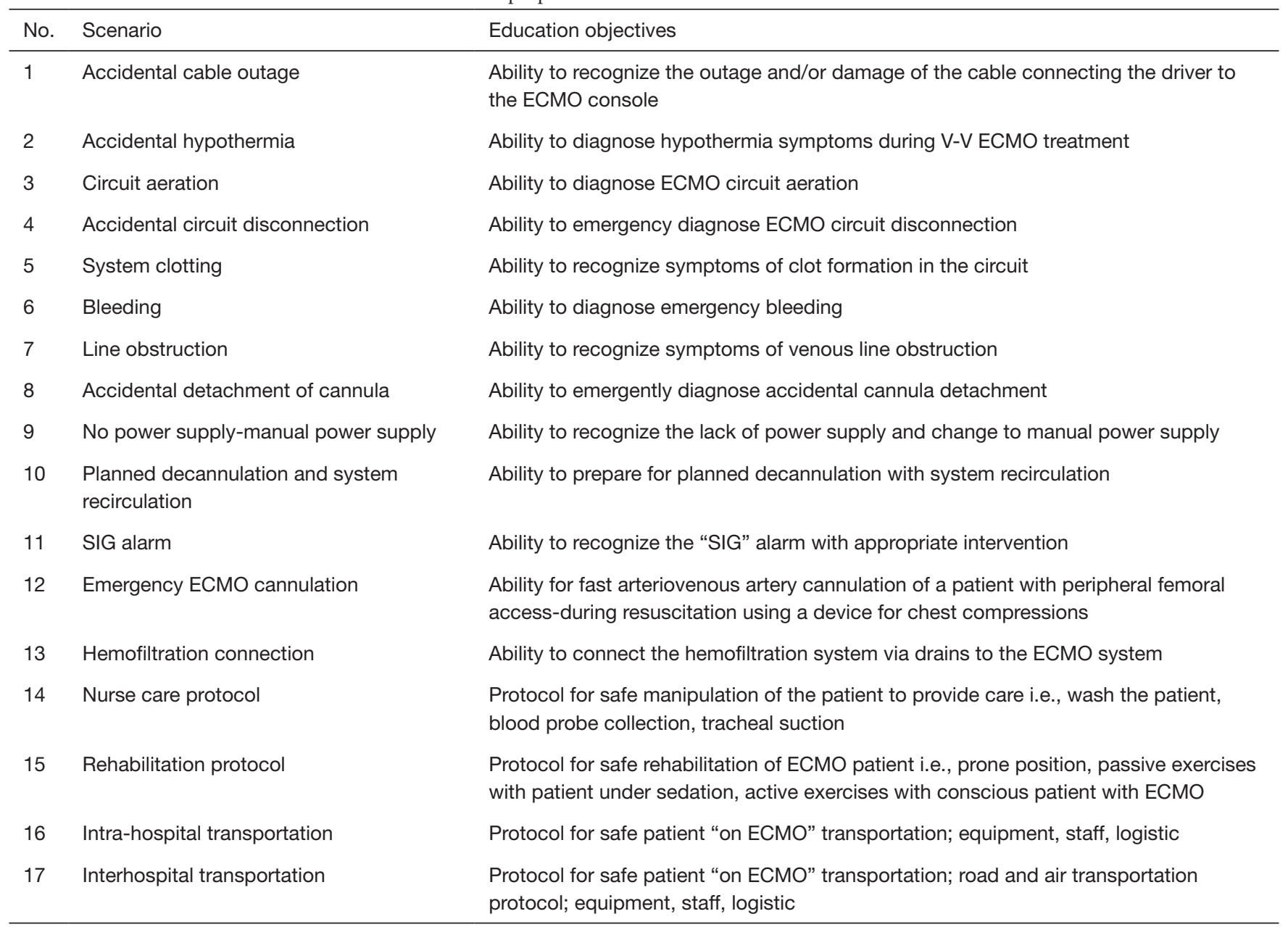

ECMO, extracorporeal membrane oxygenation.

which includes: hospital identification (Regional Department of Intensive Care): inclusion and exclusion criteria, ECMO team transport $(80 \mathrm{~km})$, therapy confirmation, veno-venous cannulation for the initiation of perfusion therapy, return transport $(80 \mathrm{~km})$ with ECMO to another hospital in a provincial city (Clinical Department of Intensive Care), where the veno-venous ECMO therapy was continued for the next 48 hours.

* "ECMO for hypothermia" which includes: prehospital identification, CPR ALS with ACC, specialist road transport on ACC; the veno-arterial (VA) cannulation and perfusion for hypothermia treatment.

The primary objectives of the scenarios include:

* Ability to diagnose and treat medical problems during ECMO treatment using clinical signs, echo or ultrasound examinations, gasometry and other necessary laboratory blood interpretations.

* Ability to effectively communicate with other members of the ECMO team.

* Adequately describe and interpret changes in clinical parameters and changes in vital signs of the patient on ECMO.

* And finally, to realize any clinical deterioration and initiate adequate treatment in order to improve patient outcome.

Our simulation team is interdisciplinary and consists of physicians experienced in VV and VA ECMO therapies, ultrasound imaging as well as percutaneous and surgical vascular access and experienced perfusionists. The team of these people composed real ECMO team and mobile ECMO team. It is supplemented by paramedics and 
specialists educators in medical simulation and an engineer responsible for the development process of the prototype.

The patient simulator was programmed with scenarios mimicking typical clinical situations (hypothermia, hypovolemia, oxygenator failure, etc.) that require prompt interventions of all trainees. The interdisciplinary workshop supervised by two staff nurses and two physicians included 16 hours in 2 days and were preceded by a mandatory e-learning course with the pre-test. The participants were naive to ECMO technology. The course was evaluated by an anonymous survey filled by all participants.

The preliminary workshops were evaluated extremely enthusiastic. All responders supported the concept of an on-line pre-test as prerequisite of entering the workshop. All the participants declared that this course would help in introducing ECMO therapy in the ICU. Moreover, they appreciated particularly the hands-on experience during that workshops, high-fidelity clinical scenarios as well as cannulation training. In the final survey "in situ" simulations were particularly highly rated. These simulations confined 48 hours of extended "ECMO for RRF" course which became a kind of training platform in Intensive Care Department.

\section{Discussion}

ECMO therapy requires a multidisciplinary team with varying clinical competences in three key areas: quality assurance, technical knowledge and behavioral skills. The most common and well-accepted educational tool is simulation based training, which provides education and training in both, clinical and non-clinical skills (10-12). Since 2006 (13), multiple variants of simulation have been used to enhance ECMO education including simple task trainers, virtual patients, standardized patients, human patient simulators, and a hybrid of these models. High-fidelity ECMO simulation has been successfully implemented in many clinical centers with noticeable impact on required competency in ECMO care. In principle, the advanced high-fidelity simulator allows unlimited possibilities in creating different clinical scenarios, especially implementation, conducting perfusion and patient transport using ECMO $(14,15)$. The technical aspects of routine and emergent care can be rehearsed and mastered, but simulation as an educational tool is still invaluable. An important advantage of regular simulation training might be time sparing effect. However, Anderson et al. (10) previously reported that simulation based ECMO training did not show any time benefit. This might be controversial, but all participants were well experienced ECMO specialists, and consequently time-sparing benefit might be limited. In the setting of a newly introduced ECMO program like the "ECMO for greater Poland", individual knowledge and experience by the various healthcare providers is limited. Therefore, time saving effect might most probably be assumed.

Zakhary et al. (16) prepared the first randomized controlled trial evaluating the utility of simulation in ECMO training. The authors reported that simulation-based ECMO training is more effective than traditional water-drill based training. In addition, simulation-based ECMO training improved technical, cognitive and behavioral skills compared to traditional class-room based teaching.

The Maastricht category II (donors after unsuccessful resuscitation) DCD procedures were already activated several times, resulting in two successful double kidney transplantations, for the first time in Poland (17-19). Additionally, we were able to successfully treat two hypothermic patients for the first time in the region, and initiated treatment using ECMO for reversible respiratory failure in several patients. We were also able to successfully perform an extensive (about $120 \mathrm{~km}$ lasting) road based transport with ECMO to a reference University Hospital where the patient was admitted to the Department of Intensive care and implemented specialist nurse and rehabilitation protocols. All critical scenarios were preliminarily tested by specialist ECMO teams and the teaching programs were introduced to several centers in the city of Poznan.

The general benefits of ECMO simulation training are: development of clinical skills and personal experience (without direct impact on patients); practice unusual or rare clinical scenarios; and allows to assess individual skills and experience (20). The economic result of simulationbased training is optimized by improving theoretical and practical skills which can foster critical thinking in bedside management of these medically complex patients. However, publications are lacking whether the use of simulators are associated with better clinical outcomes. Randomized clinical trials are needed in this field to define the priority of simulation-based ECMO training and its impact in clinical outcomes in ECMO centers (17).

Perfusion therapy and ECMO programs are currently not commonly used in the polish healthcare system. Our goal is to intensively train healthcare providers from all over the nation, which might be impossible without using high- 
fidelity simulation. Long-term effect will be improving quality of the healthcare system and wellbeing of the inhabitants of the country, as well as increasing the pool of potential organ transplants available.

A training platform called "National Reference Training Center for Extracorporeal Therapy" will be introduced and a multidisciplinary team will serve as the faculty. The high-quality simulation training is intended to be taught nationwide. Our ambition is to become a reference ECMO training center in Poland.

\section{Conclusions}

Implementation of this ECMO high-fidelity simulator intends to improve patient care. In absence of alternate adequate teaching programs, success of this unique training program mostly depends on creating adequate and realistic clinical scenarios and adequate education and training of all involved healthcare providers.

"ECMO of Greater Poland" is a newly introduced clinical program. High-fidelity simulation is an essential tool in educating and teaching all involved healthcare providers and finally improve patient's outcome.

\section{Limitations of our study}

It is proved that medical simulation training ensures safety and responsibility in therapy, especially in critically ill patients. The only limitation of the high-fidelity medical simulation as a creation tool seems to be only imagination and equipment imperfection. Owing to the hard work of the engineers it enables to practice all possible methods of cannulation, both surgical and percutaneous, also under ultrasound guidance. However, we are aware as clinicians that appropriate positioning of the venous cannula, either single or double-lumen, in the right atrium requires use of transthoracic and transesophageal echocardiographic imaging. This is another key and extremely difficult challenge for our team which we will try to meet in more faithful reflection of the clinical reality.

\section{Acknowledgements}

None.

\section{Footnote}

Conflicts of Interest: The authors have no conflicts of interest to declare.

\section{References}

1. Puslecki M, Ligowski M, Stefaniak S, et al. Using simulation to create a unique regional ECMO program for the greater Poland region. Qatar Med J 2017. 4th Annual ELSO-SWAC Conference 2017. Available online: http:// dx.doi.org/10.5339/qmj.2017.swacelso.79

2. Puslecki M, Kiel M, Ligowski M, et al. Customization of a patient simulator for ECMO training. Qatar Med J 2017. Available online: http://dx.doi.org/10.5339/qmj.2017. swacelso. 80

3. Czekajlo M, Dabrowska A. In situ simulation of cardiac arrest. Disaster Emerg Med J 2017;2:116-9.

4. Abelsson A. Learning through simulation. Disaster Emerg Med J 2017;2:125-8.

5. Czekajlo M, Dabrowski M. World SimChallenge - new simulation idea for students and medical teachers. Disaster Emerg Med J 2017;2:137-9.

6. Puslecki M, Ligowski M, Dabrowski M, et al. Highfidelity simulation - the first DCD-ECMO procedure in Poland. Disaster Emerg Med J 2017;2:50-2.

7. Al Disi M, Alsalemi A, Alhomsi Y, et al. Revolutionizing ECMO simulation with affordable yet high-Fidelity technology. Am J Emerg Med 2018;36:1310-2.

8. Atamanyuk I, Ghez O, Saeed I, et al. Impact of an openchest extracorporeal membrane oxygenation model for in situ simulated team training: a pilot study. Interact Cardiovasc Thorac Surg 2014;18:17-20; discussion 20.

9. Puślecki M, Ligowski $M$, Dabrowski $M$, et al. The role of simulation to support donation after circulatory death with extracorporeal membrane oxygenation (DCD-ECMO). Perfusion 2017;32:624-30.

10. Anderson JM, Boyle KB, Murphy AA, et al. Simulating extracorporeal mem- brane oxygenation emergencies to improve human performance. Simul Healthc 2006;1:220-7.

11. Anderson JM, Murphy AA, Boyle KB, et al. Simulating extracorporeal membrane oxygenation emergencies to improve human performance. Part II: Assessment of technical and behavioral skills. Simul Healthc 2006;1:228-32.

12. Chan SY, Figueroa M, Spentzas T, et al. Prospective assessment of novice learners in a simulation-based extracorporeal membrane oxygenation (ECMO) education program. Pediatr Cardiol 2013;34:543-52.

13. Fanning RM, Goldhaber-Fiebert SA, Udani AD, et al. Crisis resource management. In: Levine AI, DeMaria 
S Jr, Schwartz AD, et al. editors. The Comprehensive Textbook of Healthcare Simulation. New York: Springer, 2013:95-109.

14. Alinier G. ECMO simulation and beyond. Qatar Med J 2017. 4th Annual ELSO-SWAC Conference Proceedings 2017. Available online: http://dx.doi.org/10.5339/ qmj.2017.swacelso.59

15. Brum R, Rajani R, Gelandt E, Morgan L, et al. Simulation training for extracorporeal membrane oxygenation. Ann Card Anaesth 2015;18:185-90.

16. Zakhary BM, Kam LM, Kaufman BS, et al. The Utility of High-Fidelity Simulation for Training Critical Care Fellows in the Management of Extracorporeal Membrane Oxygenation Emergencies: A Randomized Controlled Trial. Crit Care Med 2017;45:1367-73.

Cite this article as: Puslecki $M$, Ligowski $M$, Kiel $M$, Dabrowski M, Stefaniak S, Sip M, Maciejewski A, Dabrowska A, Kiel-Puslecka I, Kłosiewicz T, Misterski M, Buczkowski P, Szarpak L, Ruetzler K, Perek B, Czekajlo M, Jemielity M. Prototype of extracorporeal membrane oxygenation (ECMO) therapy simulator used in regional ECMO program. J Thorac Dis 2018;10(8):5073-5079. doi: 10.21037/jtd.2018.07.25
17. Montero S, Combes A, Schmidt M. The extracorporeal membrane oxygenation (ECMO) high-fidelity simulator: the best complementary tool to learn the technique. J Thorac Dis 2017;9:4273-6.

18. Burton KS, Pendergrass TL, Byczkowski TL, et al. Impact of simulation-based extracorporeal membrane oxygenation training in the simulation laboratory and clinical environment. Simul Healthc 2011;6:284-91.

19. Fehr JJ, Shepard M, McBride ME, et al. Simulation-Based Assessment of ECMO Clinical Specialists. Simul Healthc 2016;11:194-9.

20. Banfi C, Bendjelid K, Giraud R. High-fidelity simulation for extracorporeal membrane oxygenation training, utile or futile? J Thorac Dis 2017;9:4283-5. 